\title{
Efficacy of Mobile Phone Technology for Managing Side Effects Associated with Chemotherapy among Cancer Patients: A Systematic Review and Meta-Analysis
}

\author{
Praveen Uppu ${ }^{1,2}$ (1) \\ M. Manickavasagam ${ }^{3}$ \\ 1 PhD Scholar, Sri Ramachandra Institute of Higher Education \& \\ Research, Chennai, India \\ 2 School of Nursing Sciences, Sharda University, Greater Noida, India \\ 3 Department of Medical Oncology, Sri Ramachandra Institute of \\ Higher Education and Research (DU), Porur, Chennai, India \\ ${ }^{4}$ Faculty of Nursing, Sri Ramachandra Institute of Higher Education \\ and Research (DU), Porur, Chennai, India \\ 5 School of Computer Science \& Engineering, Vellore Institute of \\ Technology, Vellore, Tamil Nadu, India
}

Nalini Sirala Jagadeesh ${ }^{4}$

K. Ramesh Babu ${ }^{5}$ Address for correspondence Mr. Uppu Praveen, Assistant Professor, School of Nursing Sciences and Research, Sharda University, Plot No: 32-34 Knowledge Park-III, Greater Noida, Uttar Pradesh, 201310, India (e-mail: uppupraveen@yahoo.com; praveenuppu@sriramachandra.edu.in).

Ind J Med Paediatr Oncol 2021;42:518-527.

\section{Abstract \\ Keywords \\ - chemotherapy \\ - side-effects \\ - mobile apps/smartphone applications \\ - cancer patient}

Background To establish the evidence related to the efficacy of mobile phone technology for managing side effects of chemotherapy and improved quality of life among patients with cancer.

Methods Articles published in peer-reviewed journals were included in this review. Randomized control trials (RCTs) and non-randomized control trials (non-RCTs) consisting of mobile-based interventions (mobile application, smart phone App-based interventions or guidelines to manage side-effects of chemotherapy or mobile health services), and adult cancer patients (aged 18 or above years) as participants who were undergoing chemotherapy and received mobile phone-based interventions as an interventional group versus control/comparator group who were getting routine or usual care were included in this systematic review. Databases such as Scopus, Science Direct, Cochrane library, PubMed, and Google Scholar were systematically searched between 2007 and 2020. Using the Cochrane risk of bias tool, the methodological quality of the included studies was evaluated by two independent authors.

Results We included 10 trials, involving 1467 cancer patients and the number of participants ranged from 50 to 457 . All trials measured the side effects of chemotherapy as the main outcome and three trials measured the quality of life as the main outcome.

Ten trials included for narrative synthesis showed a significant decrease in chemotherapy side effects and considerable improvement in the quality of life in the interventional group than in the comparison group. Meta-analysis of four RCTs containing 803 subjects concluded a significant improvement $(p<0.0001)$ in the quality of life.
DOI https://doi.org/ 10.1055/s-0041-1740120. ISSN 0971-5851. (c) 2021. Indian Society of Medical and Paediatric Oncology. All rights reserved.

This is an open access article published by Thieme under the terms of the Creative Commons Attribution-NonDerivative-NonCommercial-License, permitting copying and reproduction so long as the original work is given appropriate credit. Contents may not be used for commercial purposes, or adapted, remixed, transformed or built upon. (https://creativecommons.org/ licenses/by-nc-nd/4.0/)

Thieme Medical and Scientific Publishers Pvt. Ltd., A-12, 2nd Floor, Sector 2, Noida-201301 UP, India 
A significant improvement in the quality of life was revealed by random effects model $(\mathrm{SMD}=0.31,95 \% \mathrm{Cl}: 0.17,-0.46)$ and a significant difference $(\mathrm{Z}=4.37, p<0.001)$ was identified between experimental and control groups.

Conclusion Current review strengthens the evidence that utilizing mobile-phone based technology has favorable effects on improving the quality of life by minimizing side-effects associated with chemotherapy among cancer patients.

\section{Introduction}

In many countries, mobile-phone based technology has become an integral part of healthcare system to render health services to the needy in the form of mobile health (mHealth), smartphone apps, or applications. ${ }^{1,2}$ Globally, cancer is accountable for around 10 million deaths in $2020 .^{3}$ There are different treatment modalities for cancer, and chemotherapy is one of the most commonly used treatment of choice for cancer. ${ }^{4}$ The nature of chemotherapy is to kill the cancer cells along with healthy cells, which results in the development of certain side effects. ${ }^{5,6}$

The most common side-effects of chemotherapy include nausea, vomiting, fatigue, diarrhea, lack of sleep, loss of appetite, pain, and hair loss. ${ }^{7,8}$ The quality of life among cancer patients is affected by these side effects and may lead to psychological distress. ${ }^{6}$

Utilization of mobile-phone based technology has been increasing in the healthcare sector. ${ }^{9,10}$ Lack of awareness on management of these side-effects at home is one of the challenging issues for cancer patients. ${ }^{11}$ This issue can be resolved with the help of mobile-based technology as the mobile phones/smart phones have become a vital entity in our life. The mobile-based technology, such as smart phone apps/mHealth services, can provide information in the form of guidelines or instructions or education to the cancer patients to manage chemotherapy-associated sideeffects. $^{12,13}$

A review conducted in 2018 recommended the need to develop more comprehensive interventions through mobile technology to meet the patients' needs in the form of guidelines and self-monitoring of side effects associated with chemotherapy. $^{14}$

Use of mobile technology for self-care monitoring and reporting of the symptoms along with alert system that focus on severity of symptoms can enhance the living quality of cancer patients by reducing symptom burden of the chemotherapy. ${ }^{15}$

As per the background information and our knowledge, there are no existing meta-analyses on the intervention of utilizing mobile phone technology and its efficiency to heighten the living quality among cancer patients.

Results of this review will give intuition and support for the development of novel mobile-based interventions in the form of mobile apps/applications for progressing the quality of life. This review was decided to strengthen the evidence producing the evidence on determining the clinical out- comes of the patient's using mobile phone technology. The objective of this review is to establish the evidence related to the efficacy of mobile phone technology for managing side effects of chemotherapy and improving the quality of life among cancer patients.

\section{Methods}

The authors followed Joanna Briggs Institute Manual for Evidence Synthesis (Guidance for authors to conducting systematic reviews) $)^{16}$ and the PRISMA guidelines for the preparation of this systematic review and meta-analysis. ${ }^{17}$

\section{Eligibility Criteria Inclusion criteria}

a) Articles published in peer-reviewed journals.

b) Study designs: randomized control trials (RCTs) and non-randomized control trials (non-RCTs).

c) Interventions: Studies consisting of mobile-based interventions (mobile application, smart phone Appbased interventions or guidelines to manage sideeffects of chemotherapy or mHealth services.

d) Participants: The trials that included adult cancer patients (aged 18 or above years) who were undergoing chemotherapy, and received mobile phone-based interventions versus control/comparator group who were getting routine or usual care, across all types of cancers, gender, race, regions, and country.

e) Settings: Conducted in rural or urban areas or Hospitals or oncology units or clinical settings.

f) Outcomes: Studies were included if they described either a few or all side effects of chemotherapy such as nausea, vomiting, pain, mucositis, fatigue, sleep disturbances, diarrhea, constipation, dyspnea, urinary problems, hand foot syndrome, hair loss, and poor quality of life.

g) Language: Trials published in English language only.

\section{Exclusion Criteria}

a) Conference abstracts, databases containing only abstracts, books, and gray literature were excluded.

\section{Information Sources}

The databases such as Science Direct, Scopus, Cochrane Library, PubMed, and Google Scholar were searched for the eligible trials reported between 2007 and 2020. In addition to this, hand search of references was performed 
from related trials to identify the studies based on inclusion criteria.

\section{Search Strategy}

The search strategy was developed comprehensively using keywords in congruence with PICO terms (population, intervention, comparator, and outcome) to identify the relevant studies by following keywords: "Cancer patients" AND chemotherapy AND "mobile application" OR "Mobile apps" OR mHealth AND "side effects" OR "symptom management" AND "quality of life."

The title and abstracts screening of the retrieved studies was done based as per the inclusion criteria. Duplicate trials were removed by screening the title and abstracts using Reference Manager (Zotero). The two authors (UP and SJN) independently performed screening of the retrieved abstracts based on pre-determined inclusion criteria, followed by screening of full-text articles.

\section{Data Collection Process}

Quantitative data of the included studies were extracted independently by two authors based on JBI experimental studies data extraction tool. ${ }^{16}$ The data extraction form consisted of details such as author, year of publication, location, study type, sample size/group, participants type and age, intervention details and duration, outcome measures, instruments used and study findings.

\section{Data Items}

a. Participants: The trials that included adult cancer patients (aged 18 or above years) who were undergoing chemotherapy, and received mobile phone-based interventions versus control/comparator group who are under routine or usual care.

b. Intervention: The experimental group cancer patients had received interventions based on mobile technology. Trials exploring the effectiveness of mobile-based interventions (mobile application, smart phone App-based interventions or guidelines to manage side effects of chemotherapy or mHealth services) on cancer patients undergoing chemotherapy were included.

c. Comparison: The comparator group included cancer patients undergoing chemotherapy and who received regular or routine care.

d. Outcome: In this systematic review, we analyze the sideeffects of chemotherapy such as nausea, fatigue, vomiting, oral mucositis, depression, numbness, anxiety, hair loss, diarrhea. and quality of life. Meta-analysis was done for the quality of life.

\section{Study Risk of Bias Assessment and Effect Measures}

Using the Cochrane risk of bias tool, the methodological quality of the included studies was evaluated by two independent authors (UP and SJN). It included six domains as mentioned in -Fig. 1. We found a $100 \%$ low-risk bias regarding random sequence generation, and $75 \%$ unclear bias was noted toward allocation concealment. The details

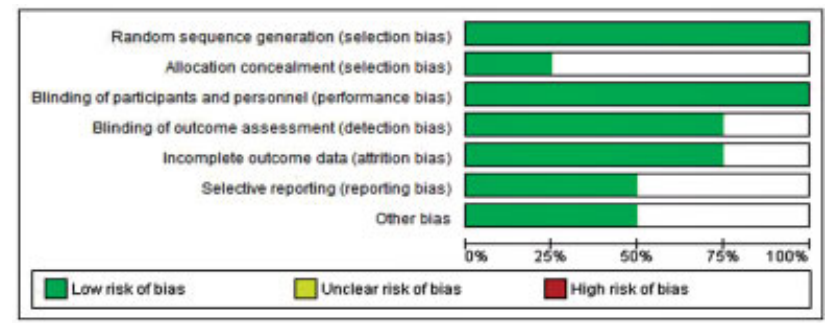

Fig. 1 Risk of bias chart based on review authors' judgment.

regarding the percentages of risk of bias across all trials are reported in - Fig. 1 .

\section{Outcome of the Search}

A total of 3,647 studies were found through electronic databases. Eight duplicated were removed using Reference Manager. After screening the title and abstracts, 3,622 studies were omitted as they did not meet the criteria of this review as per the PICO. Full-text articles assessed for the eligibility were 17 , from which 7 articles were eliminated as they were unable to fulfil the inclusion criteria. The rational to exclude the full-text articles along with study selection and elimination process is mentioned in the PRSIMA flow chart (-Fig. 2). Finally, 10 articles were involved for qualitative narrative synthesis, of which 4 RCTs were included for meta-analysis on the variable "quality of life."

\section{Synthesis}

The outcome measures of the trials that included chemotherapy-associated side-effects and quality of life between cancer patients who received mobile phone-based technology as intervention and the control group who were on routine care were differentiated. A meta-analysis on the quality of life was conducted to pool the results of RCTs using the Rev-Man v5.4 software. The intervention's effect size for the quality of life was estimated as a continuous outcome by pooling the standardized mean difference applying a random-effect model with $95 \%$ confidence interval (CI). $I^{2}$ value was used to analyze the heterogeneity in the included trials. The quality evidence and the strength of the outcome (effect sizes: 0.8 and above-large, around 0.5 medium, around 0.2-small) were measured based on the GRADE approach guidelines. ${ }^{18,19}$ The findings are reported in -Table 1.

\section{Results}

\section{Characteristics of Patients}

In this systematic review, 10 clinical trials involved 1,467 cancer patients and the number of participants ranged from 50 to 457 . The mean age of the subjects was 60 years and both males and females were included, except for four RCTs, where the participants were only females. ${ }^{20-23}$ All trials measured the side effects of chemotherapy as the main outcome and three trials measured the quality of life as the main outcome. In all trials, the interventional group received mobile-based technology as an intervention and 


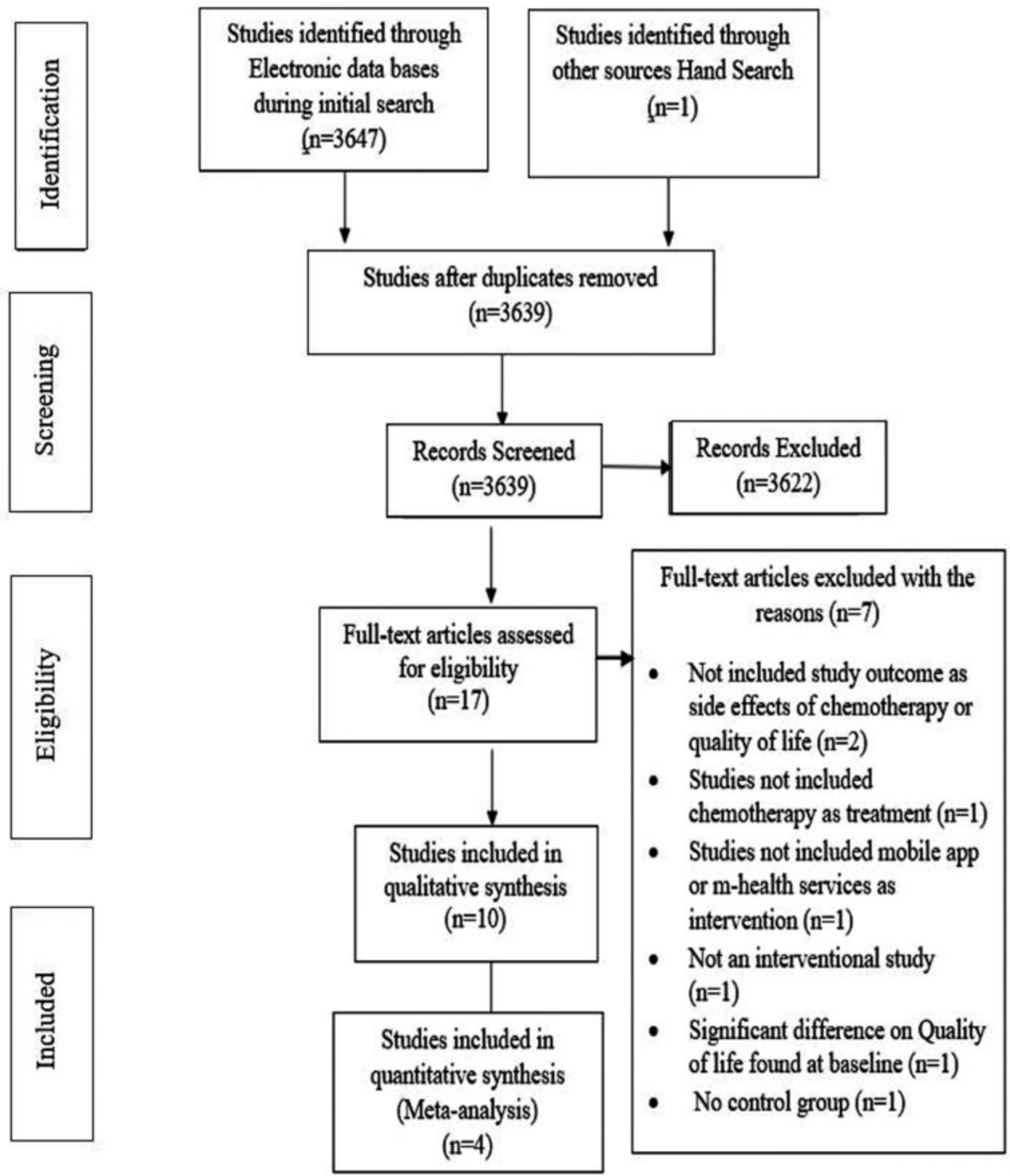

Fig. 2 Study selection process based on the PRISMA flow chart.

the control group was under routine/usual care. The details are mentioned in - Table 2.

Effects of intervention: The quality of life was analyzed as a major outcome in this meta-analysis. The effectiveness of mobile-based technology interventions was calculated based upon the difference between intervention group and control group post test scores.

A total of 10 trials were included for narrative synthesis that showed a significant decrease in symptoms/side effects and improved quality of life in the interventional group than in the comparison group. ${ }^{11,13,20-27}$

\section{Statistical Analysis}

The meta-analysis of four RCTs containing 803 cancer subjects concluded that a significant improvement $(p<0.0001)$ in the quality of life.

It shows that the mobile phone-based technology interventions were effective in enhancing the cancer patient's quality of life. A significant improvement in the quality of life was revealed by random effects model using standardized mean difference (SMD $=0.31,95 \% \mathrm{Cl}: 0.17,-0.46)$ and a significant difference $(Z=4.37, p<0.001)$ was identified between experimental and control groups. The pooled 


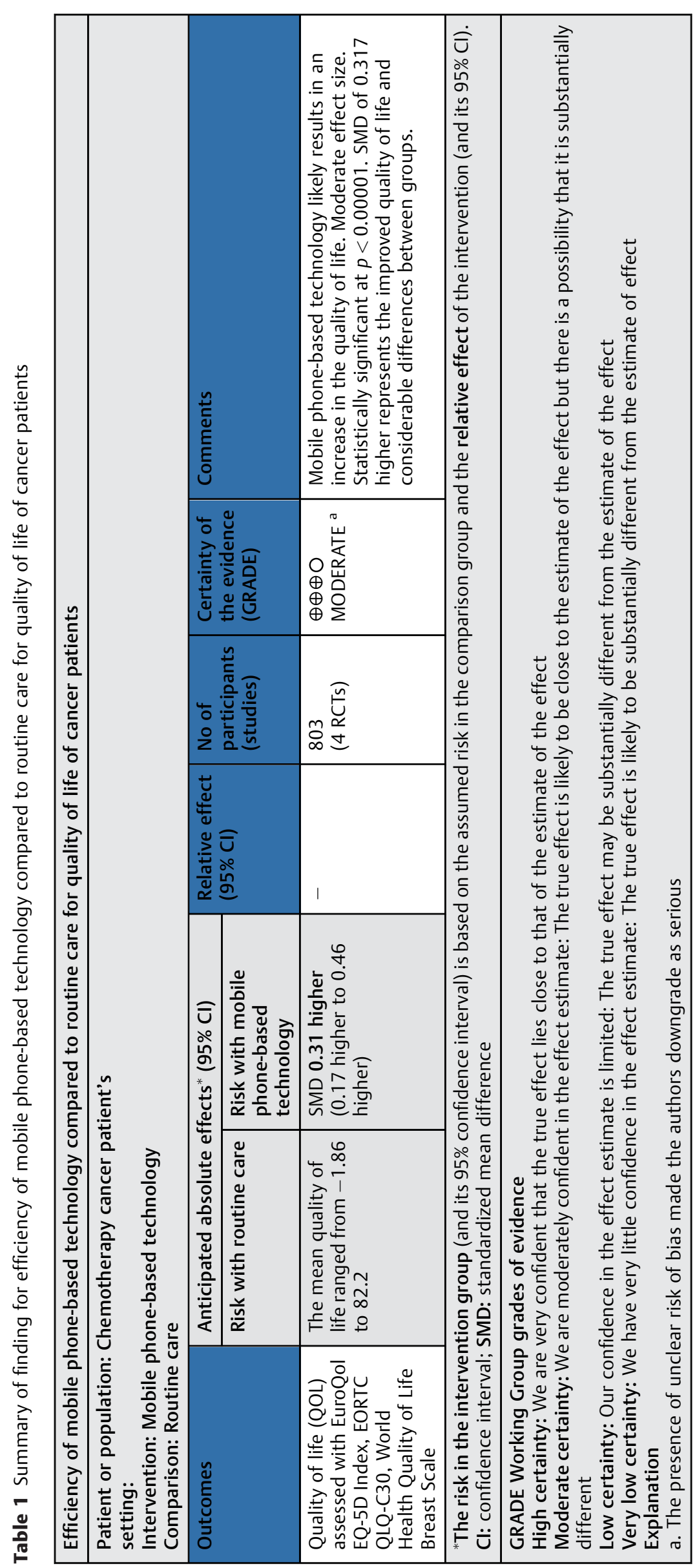




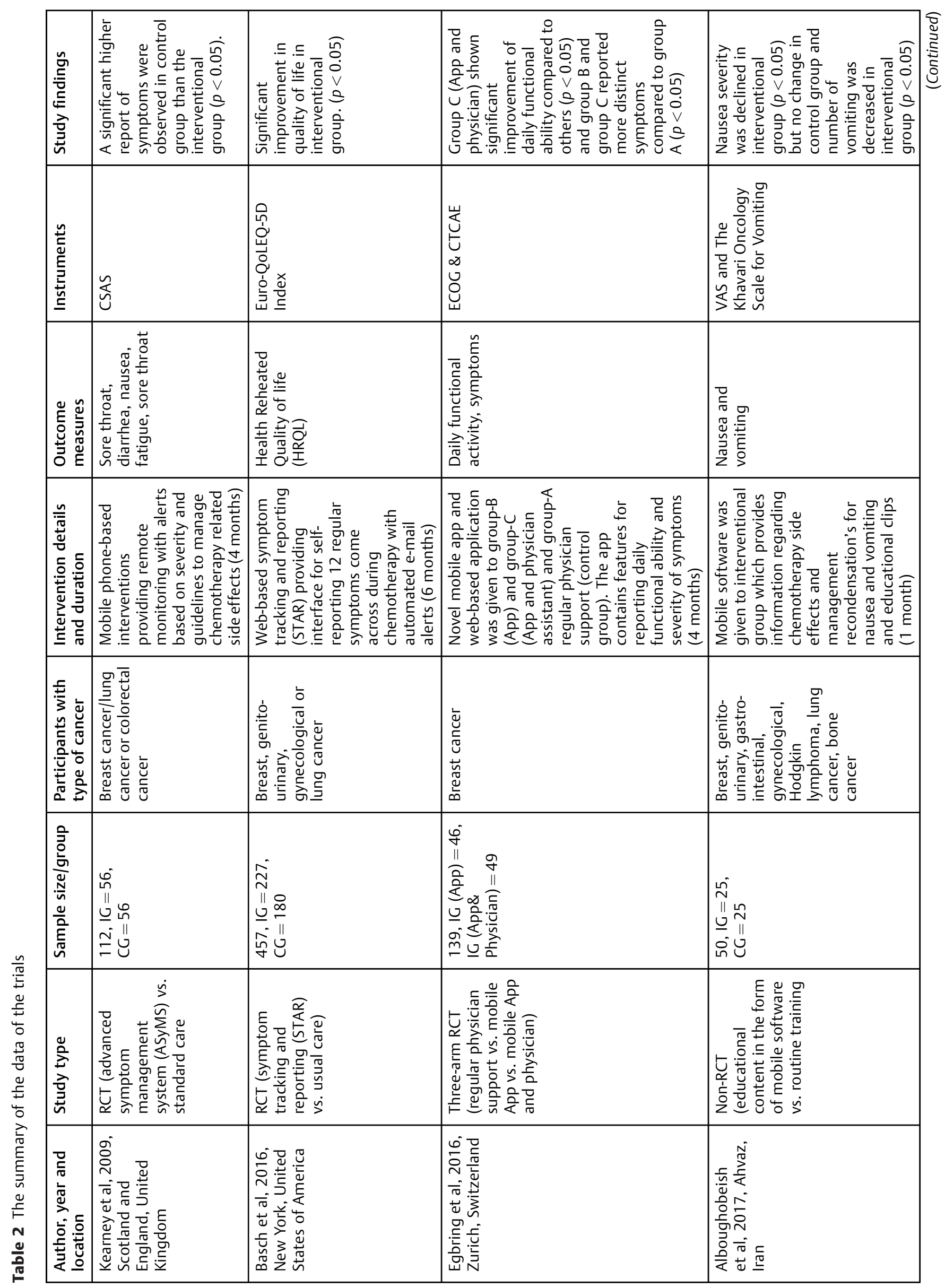




\begin{tabular}{|c|c|c|c|c|}
\hline 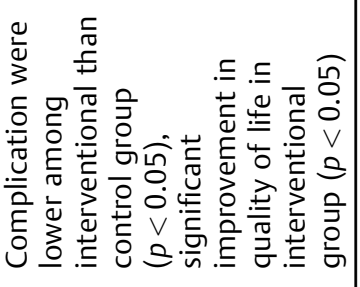 & 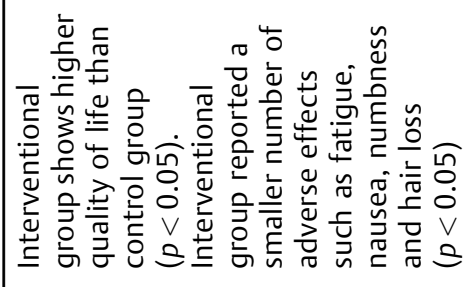 & 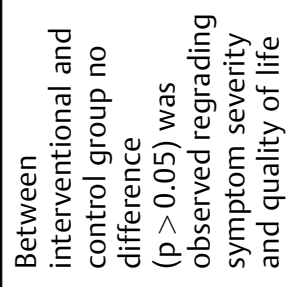 & 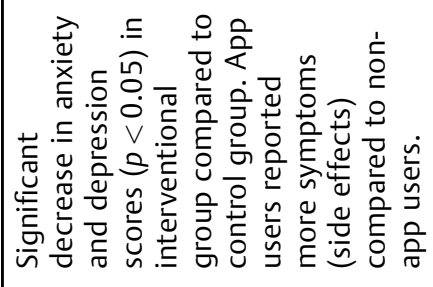 & 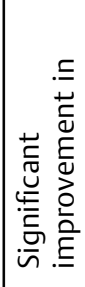 \\
\hline & 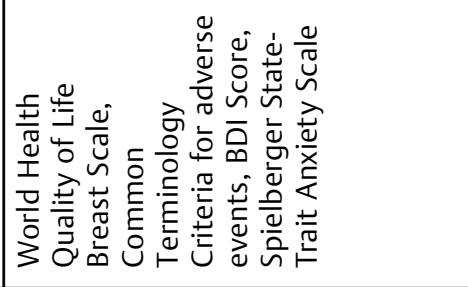 & 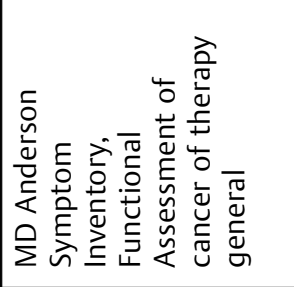 & 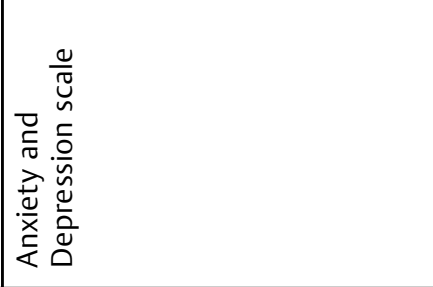 & 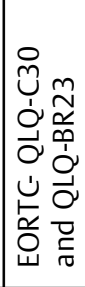 \\
\hline 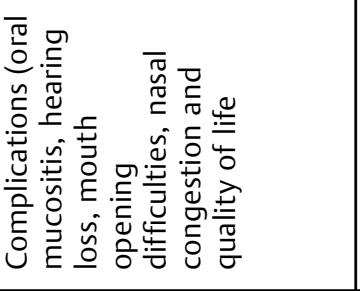 & 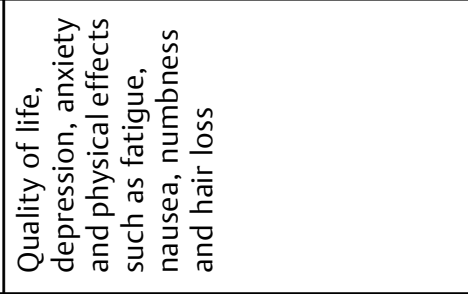 & 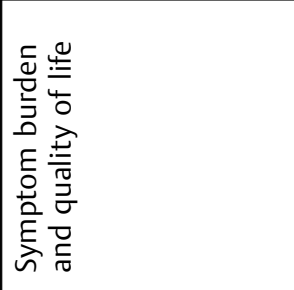 & 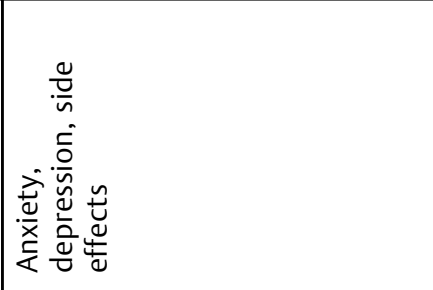 & 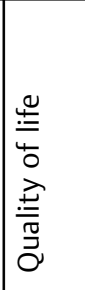 \\
\hline 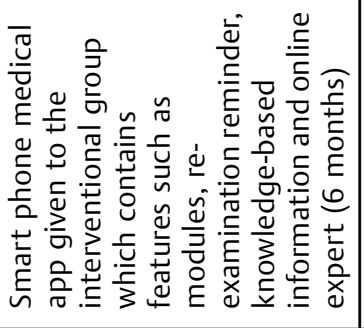 & 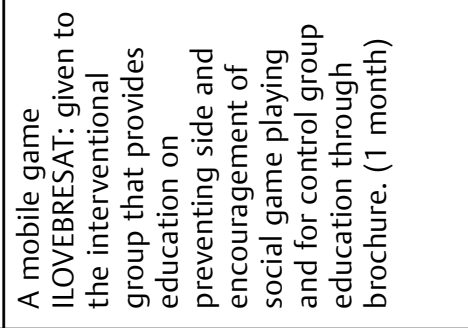 & 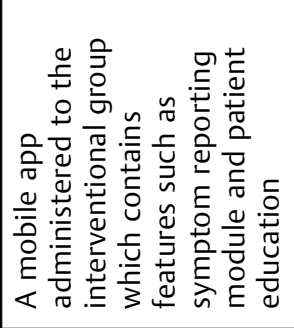 & 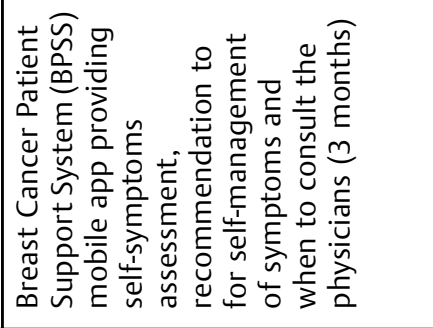 & 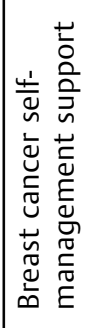 \\
\hline 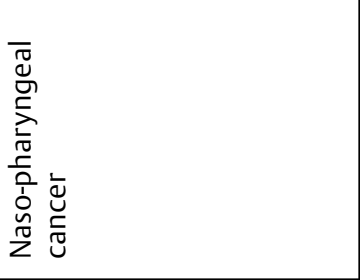 & 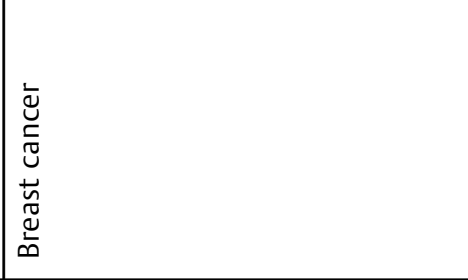 & 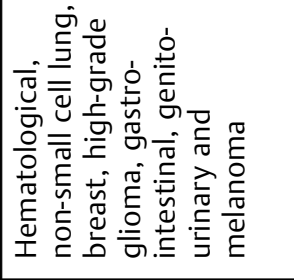 & 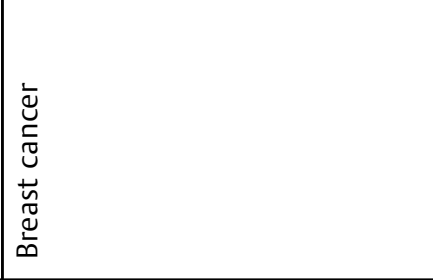 & 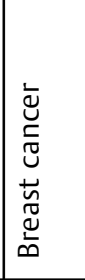 \\
\hline 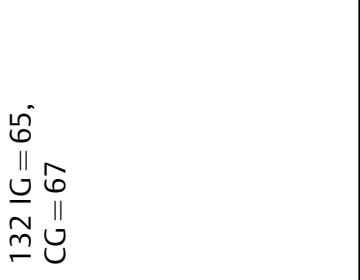 & 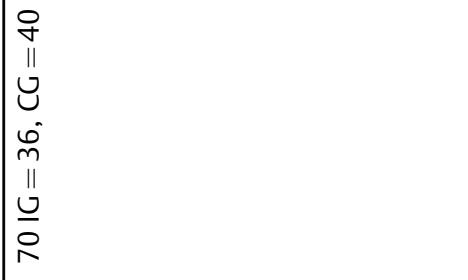 & 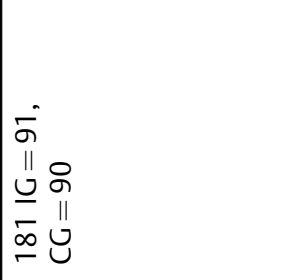 & 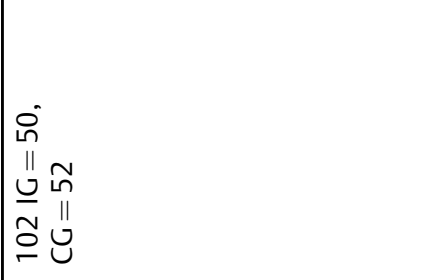 & 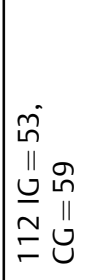 \\
\hline 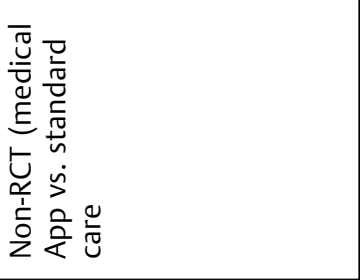 & 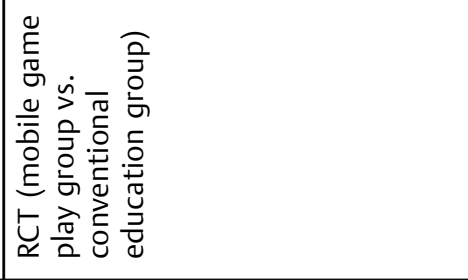 & 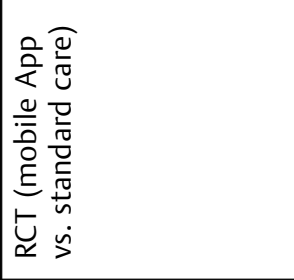 & 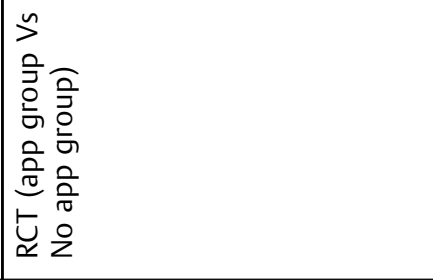 & \\
\hline 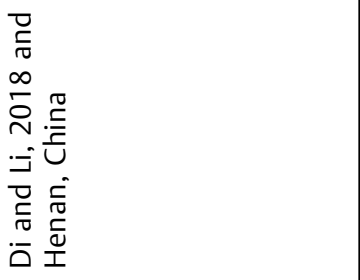 & 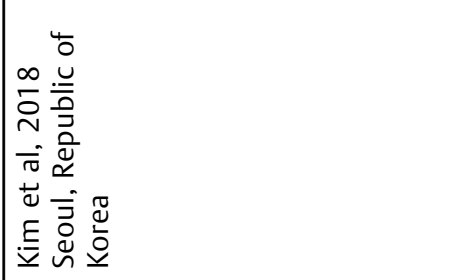 & 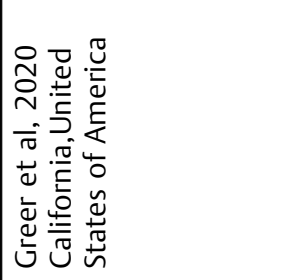 & 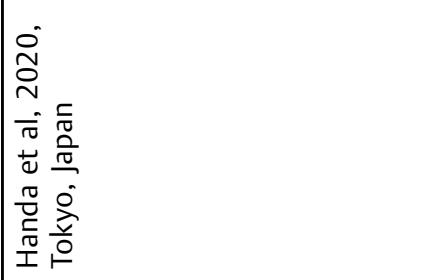 & 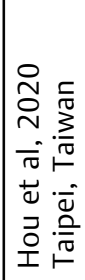 \\
\hline
\end{tabular}




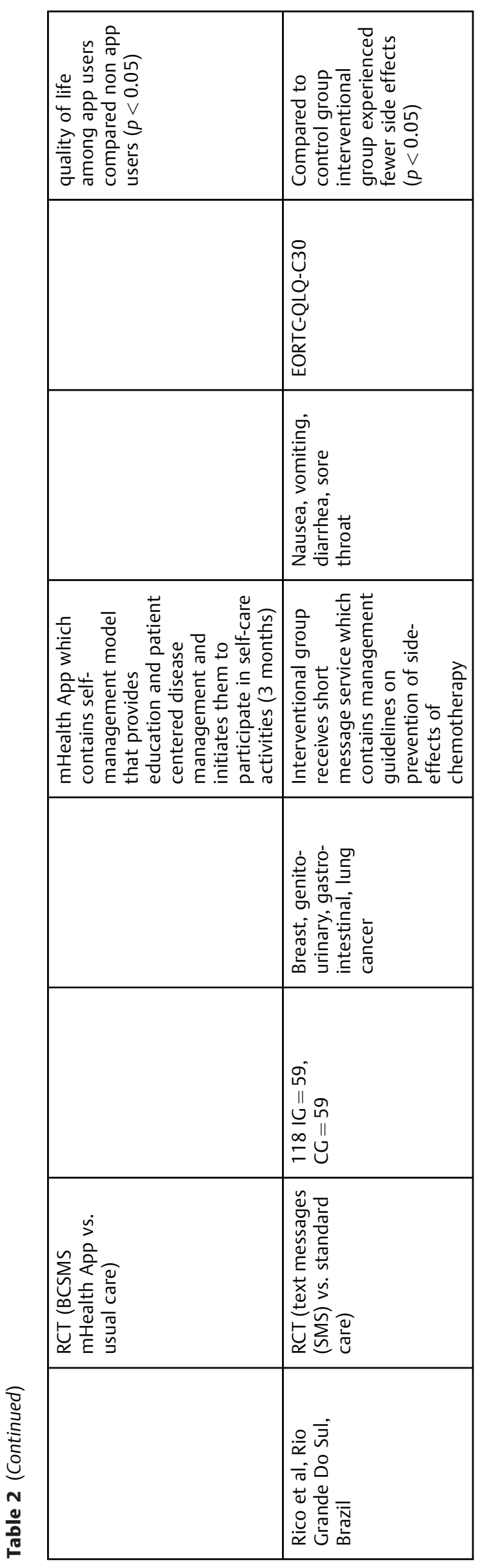

studies were homogenous ( $p=0.46, I^{2}=0 \%$ ). Because the four RCTs used different tools for measuring the quality of life, the standardized mean difference with random effects model was used for effect measure (-Fig. 3 ).

\section{Publication Bias}

Publication bias was not evaluated as only four trials were incorporated for this meta-analysis.

\section{Evidence of Quality}

There was a moderate quality of evidence for the assessed quality of life due to an unclear risk of bias (-Table 1, -Fig. 1).

\section{Discussion}

This systematic review gives evidence that mobile phone technology interventions (mobile applications, smart phone App-based interventions, or guidelines to handle the complications of chemotherapy or mHealth services) reduced the side effects/symptoms and improved the chemotherapy cancer patient's quality of well-being.

Our review included 10 trials published between 2007 and 2020, identified all studies reporting that chemotherapy-associated side-effects were reduced and enhanced the quality of life. All the studies showed favorable outcomes.

Four trials were incorporated in this meta-analysis to evaluate the effectiveness of mobile phone technology interventions among cancer patients regarding the changes in the quality of life. Out of four, two trials revealed significant favorable change in the quality of life, another two trials showed improvement in the quality of life but results were not significant. However, the pooled result of all four trials showed that the mobile phone technology interventions significantly improved the quality of life.

This systematic review identified that physical effects such as nausea, vomiting, diarrhea, sore throat (oral mucositis) fatigue, and psychological effects such as depression and anxiety were the most common side-effects experienced by the cancer patients under chemotherapy. These findings are congruent with those of previous studies that highlighted the common side effects experienced by the cancer patients under chemotherapy. ${ }^{5,6}$

The outcome of our systematic review was supported with the previous systematic reviews that highlighted that mobile phone technology interventions had helped to minimize chemotherapy complications and improved cancer patient's quality of life. 7,14

The findings of this review will encourage healthcare professionals and organizations to develop innovative methods using mobile phone technology that can help cancer patients to manage the side effects associated with chemotherapy by themselves in their respective home settings. However, mobile phone-based technology alone may not enhance the well-being of cancer patients. The outcome of this review might disburse the opportunity for the usage of mobile phone-based technology for rendering the healthcare services to the cancer patients. 


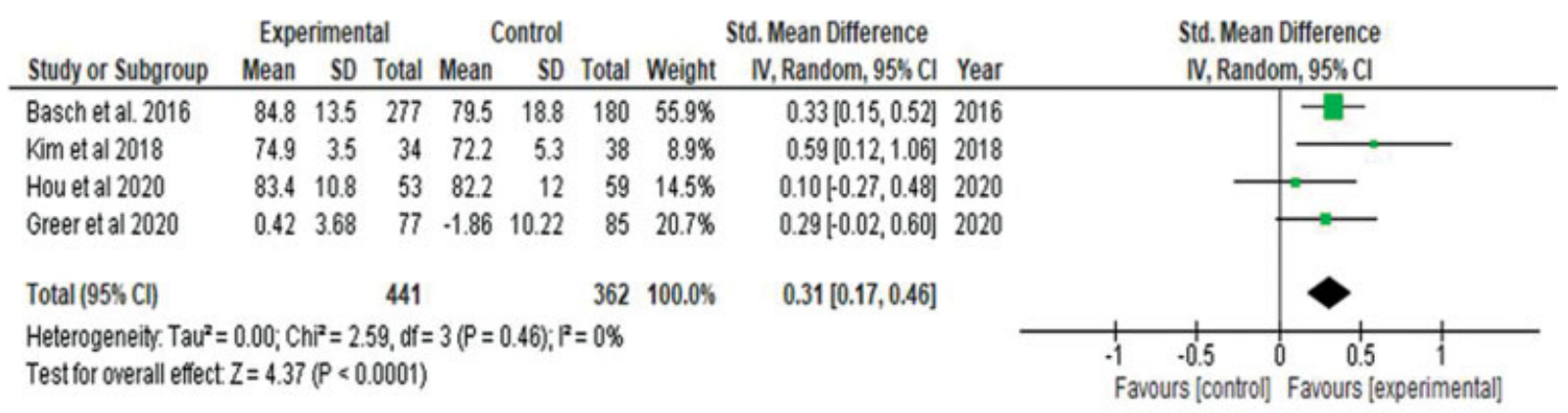

Fig. 3 Effectiveness of mobile phone-based technology on the quality of life.

\section{Limitations}

Trials published in English language only were included for this systematic review, thereby raising the chances for language bias. Publication bias was not assessed due to restricted number of trials in the meta-analysis.

\section{Conclusion}

The evidence of this systematic review and meta-analysis supported that mobile phone-based interventions help to improve the quality of life by minimizing the side-effects associated with chemotherapy among cancer patients. As there is a rapid growth of using mobile phone/smart phones by everyone, incorporating mobile phone technology in delivering the health services is becoming very easy. However, caution needs to be taken in guiding the users about the proper way of utilizing the technology-based services. There is a need for further high quality of evidence through trials for effective implementation of mobile-based technology services for cancer patients.

Systematic Review Registration No

International Prospective Register for Systematic Reviews

(Prospero Registration No-CRD42020152520).

Conflict of Interest -

None declared.

\section{Acknowledgment}

I would like to acknowledge my dear friend Ms. Tenzin Fagdol, Ph.D. Scholar, Manipal College of Nursing, Manipal Academy of Higher Education, Manipal, Udupi, Karnataka for her help in searching studies from databases.

\section{References}

1 Uddin J, Biswas T, Adhikary G, et al. Impact of mobile phone-based technology to improve health, population and nutrition services in rural Bangladesh: a study protocol. BMC Med Inform Decis Mak 2017;17(01):101

2 Akter S, Ray P. mHealth - an Ultimate Platform to Serve the Unserved. Yearb Med Inform 2010:94-100

3 Cancer [Internet]. [Cited 2021 Jan 21]. Accessed October 18, 2021 from: https://www.who.int/news-room/fact-sheets/detail/cancer
4 Treatment Modalities of Cancer | Cancer Treatment Methods Cytecare [Internet]. Cytecare Hospital in Bangalore. 2019 [cited 2021 Jan 21]. Available from: https://cytecare.com/blog/treatment-modalities-of-cancer/

5 Chan H-K, Ismail S. Side effects of chemotherapy among cancer patients in a Malaysian General Hospital: experiences, perceptions and informational needs from clinical pharmacists. Asian Pac J Cancer Prev 2014;15(13):5305-5309

6 Pearce A, Haas M, Viney R, et al. Incidence and severity of self-reported chemotherapy side effects in routine care: a prospective cohort study. Ganti AK, editor. PLoS One 2017;12(10):e0184360. Available at https:// www.ncbi.nlm.nih.gov/pmc/articles/PMC5634543/. Accessed November 22, 2021

7 Weaver A, Young AM, Rowntree J, et al. Application of mobile phone technology for managing chemotherapy-associated sideeffects. Ann Oncol 2007;18(11):1887-1892

8 Brant JM, Beck SL, Dudley WN, Cobb P, Pepper G, Miaskowski C. Symptom trajectories during chemotherapy in outpatients with lung cancer colorectal cancer, or lymphoma. Eur J Oncol Nurs 2011;15(05):470-477

9 Davoodi S, Safdari R, Ghazisaeidi M, Mohammadzadeh Z, Azadmanjir Z. Prevention and early detection of occupational cancers a view of information technology solutions. Asian Pac J Cancer Prev 2015;16(14):5607-5611

10 Agboola S, Flanagan C, Searl M, Elfiky A, Kvedar J, Jethwani K. Improving outcomes in cancer patients on oral anti-cancer medications using a novel mobile phone-based intervention: study design of a randomized controlled trial. JMIR Res Protoc 2014;3 (04):e79

11 Rico TM, Dos Santos Machado K, Fernandes VP, et al. Use of text messaging (SMS) for the management of side effects in cancer patients undergoing chemotherapy treatment: a randomized controlled trial. J Med Syst 2020;44(11):193

12 Osterberg L, Blaschke T. Adherence to medication. N Engl J Med 2005;353(05):487-497

13 Egbring M, Far E, Roos M, et al. A mobile app to stabilize daily functional activity of breast cancer patients in collaboration with the physician: a randomized controlled clinical trial. J Med Internet Res 2016;18(09):e238

14 Richards R, Kinnersley P, Brain K, McCutchan G, Staffurth J, Wood F. Use of mobile devices to help cancer patients meet their information needs in non-inpatient settings: systematic review. JMIR Mhealth Uhealth 2018;6(12):e10026

15 Crafoord M-T, Fjell M, Sundberg K, Nilsson M, Langius-Eklöf A. Engagement in an interactive App for symptom self-management during treatment in patients with breast or prostate cancer: mixed methods study. J Med Internet Res 2020;22(08):e17058

16 Aromataris E, Munn Z. Joanna Briggs Institute Manual for Evidence synthesis. JBI; 2020

17 Moher D, Liberati A, Tetzlaff J, Altman DGPRISMA Group. Preferred reporting items for systematic reviews and meta-analyses: the PRISMA statement. PLoS Med 2009;6(07):e1000097 
18 Guyatt GH, Thorlund K, Oxman AD, et al. GRADE guidelines: 13. Preparing summary of findings tables and evidence profilescontinuous outcomes. J Clin Epidemiol 2013;66(02):173-183

19 GRADEpro GDT. GRADEpro Guideline Development Tool [Software]. McMaster University; 2015 (developed by Evidence Prime, Inc.). Available from gradepro.org

20 Alboughobeish SZ, Asadizaker M, Rokhafrooz D, Cheraghian B. The effect of mobile-based patient education on nausea and vomiting of patients undergoing chemotherapy. Biomed Res 2017;28(19)

21 Basch E, Deal AM, Kris MG, et al. Symptom monitoring with patient-reported outcomes during routine cancer treatment: a randomized controlled trial. J Clin Oncol 2016;34(06):557-565

22 Di R, Li G. Use of a smartphone medical app improves complications and quality of life in patients with nasopharyngeal carcinoma who underwent radiotherapy and chemotherapy. Med Sci Monit 2018;24:6151-6156

23 Greer JA, Jacobs JM, Pensak N, et al. Randomized trial of a smartphone mobile app to improve symptoms and adherence to oral therapy for cancer. J Natl Compr Canc Netw 2020;18(02): 133-141

24 Handa S, Okuyama H, Yamamoto H, Nakamura S, Kato Y. Effectiveness of a smartphone application as a support tool for patients undergoing breast cancer chemotherapy: a randomized controlled trial. Clin Breast Cancer 2020;20(03): 201-208

25 Hou IC, Lin HY, Shen SH, et al. Quality of life of women after a first diagnosis of breast cancer using a self-management support mHealth App in Taiwan: randomized controlled trial. JMIR Mhealth Uhealth 2020;8(03):e17084

26 Kearney N, McCann L, Norrie J, et al. Evaluation of a mobile phonebased, advanced symptom management system (ASyMS) in the management of chemotherapy-related toxicity. Support Care Cancer 2009;17(04):437-444

27 Kim HJ, Kim SM, Shin H, Jang J-S, Kim YI, Han DH. A mobile game for patients with breast cancer for chemotherapy self-management and quality-of-life improvement: randomized controlled trial. J Med Internet Res 2018;20(10):e273 